\title{
Role of chronic inflammation in the pathogenesis of nonalcoholic steatohepatitis: lessons from a unique mouse model using melanocortin receptor-deficient mice
}

\author{
Michiko Itoh ${ }^{1), 2), 3)}$, Takayoshi Suganami2),4) and Yoshihiro Ogawa ${ }^{5)}$ \\ 1) Department of Organ Network and Metabolism, Graduate School of Medical and Dental Sciences, Tokyo Medical and Dental \\ University, Tokyo, Japan \\ 2) Department of Molecular Metabolism and Medicine, Research Institute of Environmental Medicine, Nagoya University, Nagoya, \\ Japan \\ 3) On-skin Artificial Pancreas Project, Kanagawa Institute of Industrial Science and Technology, Kawasaki, Japan \\ 4) Department of Immunometabolism, Nagoya University Graduate School of Medicine, Nagoya, Japan \\ 5) Department of Medicine and Bioregulatory Science, Graduate School of Medical Sciences, Kyushu University, Fukuoka, Japan
}

\begin{abstract}
Nonalcoholic fatty liver disease (NAFLD) is a clinical spectrum that encompasses simple steatosis to nonalcoholic steatohepatitis (NASH), the latter of which is characterized by chronic inflammation and fibrosis. NASH is now becoming the leading cause of cirrhosis and hepatocellular carcinoma worldwide. The pathophysiology of NASH is multifactorial and, therefore, not yet completely understood, although it is pointed out that hepatocyte death and subsequent inflammation play a central roles in disease pathogenesis. Since stromal cells dramatically change their cellular components and activation status as liver fibrosis develops, it is important to reveal the subset responsible for the disease development in each etiology. Macrophages foam crown-like structures (CLS), in which CD11c-positive macrophages surround dead hepatocytes induced by lipotoxic injury in mouse and human NASH. Hepatic CLS-constituting macrophages exhibit gene expression profiles distinct from other scattered macrophages in the liver, suggesting NASH-specific macrophages represent a subset that drives metabolic stress-induced liver fibrosis. Moreover, cancer-associated pathways are upregulated in activated fibroblasts from the liver of a mouse NASH model, suggesting that fibroblasts provide the microenvironment that promotes tumor progression. A better understanding of the upstream signals and regulatory mechanisms that drive the generation of NASH-specific macrophage and fibroblast subsets is crucial for the development of novel diagnostic and therapeutic strategies.
\end{abstract}

Key words: Chronic inflammation, Macrophages, Fibroblasts, Cell death, Crown-like structure

\section{Introduction}

Nonalcoholic fatty liver disease (NAFLD) is recognized as a hepatic phenotype of metabolic syndrome, and is highly prevalent in subjects with obesity and diabetes [1]. NAFLD is an emerging healthcare problem worldwide in both children and adults, with an estimated global prevalence of $25 \%$ [2]. Although the clinical spectrum encompasses simple steatosis with no or minimal inflammatory activity to nonalcoholic steatohepatitis

Submitted Jan. 6, 2021; Accepted Mar. 26, 2021 as EJ21-0002

Released online in J-STAGE as advance publication May 7, 2021

Correspondence to: Michiko Itoh, Department of Molecular Metabolism and Medicine, Research Institute of Environmental Medicine, Nagoya University, Furo-cho, Chikusa-ku, Nagoya, Aichi 464-8601, Japan.

E-mail: mito@riem.nagoya-u.ac.jp
(NASH), the latter of which is characterized by chronic inflammation and fibrosis [3]. NASH is already considered among the top etiologies for hepatocellular carcinoma (HCC) and indications for liver transplantation [4]. However, not all patients progress from simple steatosis to NASH and the reasons underlying the progression remain unclear. During the course of NASH development, stromal cells dramatically change their cellular component and activation status in response to parenchymal cell (hepatocyte) injury and death [5]. For better understanding of the pathophysiology of NASH, many attempts have been made to generate rodent models of NASH. The authors have reported that melanocortin 4 receptor (MC4R)-deficient (MC4R-KO) mice sequentially develop diet-induced hepatic steatosis, liver fibrosis and HCC [6]. In this review article, we discuss recent progress in understanding the molecular mechanisms of 
NASH, especially focusing on the features of diseasespecific macrophage and fibroblast identified in our NASH model.

\section{Definition and Clinical Characteristics of NAFLD}

NAFLD is defined as fat deposition in hepatocytes $(>5 \%)$ without excessive alcohol intake or other conditions associated with steatosis, and NASH is characterized by hepatocyte injury and death (described histologically as hepatocyte ballooning) and inflammation with or without fibrosis. The natural history of NAFLD is poorly understood, because invasive liver biopsy remains the gold-standard for the diagnosis of NASH. It has been reported that $10 \%-20 \%$ of patients with NAFLD progress to NASH, and approximately $20 \%$ of NASH progress to advanced liver fibrosis with a risk of extrahepatic complications, cirrhosis, and HCC $[7,8]$. Recent data have emphasized the importance of fibrosis as the strongest determinant of HCC development and liver-related mortality [9-11]. Nonetheless, there is no accurate noninvasive method to detect NASH and treatment of this disease is limited to lifestyle modifications. The prevalence and severity of NAFLD are substantially increased in patients with type 2 diabetes, suggesting that NAFLD and type 2 diabetes share insulin resistance as a common pathophysiological mechanism $[12,13]$. However, as NAFLD is highly heterogenous in terms of their primary drivers and coexisting disease modifiers, it poses a serious impediment to the discovery of therapeutic strategies and biomarkers. Recently, a consensus group proposed that nomenclature of NAFLD should be updated to metabolic (dysfunction) associated fatty liver disease (MAFLD) to more accurately reflect current knowledge of fatty liver diseases [14]. Although the nomenclature to appropriately describe the disease's pathophysiology has not been settled, it is crucial to understand the subphenotypes and the dominant disease driver to accelerate the translational path to new treatments.

\section{Rodent Models of NAFLD and NASH}

Animal models are necessary to understand the pathogenesis of NAFLD/NASH and evaluate mechanismbased therapies (Table 1). Steatosis is readily induced by overnutrition with a high-carbohydrate or high-fat diet (HFD) feeding, whereas diet-induced obesity leads to mild inflammation and liver fibrosis only after a longterm exposure (usually longer than one year) [15-17]. Feeding animals with atherogenic diets, containing fat and cholesterol as well as cholete, results in liver damage and fibrosis [18-20]. There are concerns that very high cholesterol content is undesirable, being toxic and far above human consumption. It is widely known that methionine and choline-deficient (MCD) diets induce steatohepatitis and fibrosis in the short term ( $\sim 6$ weeks) [21-23]. However, mice on MCD diets are cachexic and insulin sensitive, and the pathological features are different from those of human NASH (lack of hepatocyte ballooning and Malloy-Denk bodies) [24]. To overcome the weight loss and insulin sensitivity caused by MCD diets, choline-deficient, amino acid-defined diets combined with HFD have been developed, which induce weight gain, steatohepatitis and mild liver fibrosis around 6 months, with progression to advanced-stage fibrosis and HCC after one year [25]. In addition to the dietary models described above, genetic models using specific gene mutant mice combined with Western diet (WD) feeding have been used [26, 27]. We have reported MC4R-KO mice fed HFD or WD as a novel rodent model of NASH [6], and this model has been reproduced in several laboratories $[28,29]$. MC4R is a seventransmembrane $\mathrm{G}$ protein-coupled receptor that is mainly expressed in the hypothalamus. It regulates food intake and energy expenditure downstream of the leptin signaling [30]. MC4R-KO mice show severe obesity and insulin resistance, sequentially exhibit typical steatohepatitis pathology and pericellular fibrosis after 20-week HFD or WD feeding, and finally develop HCC after one year [6]. Because MC4R expression is mainly restricted to the central nervous system and is undetectable in the liver, it is conceivable that the hepatic phenotype in MC4R-KO mice results from metabolic derangements induced by hyperphagic obesity. MC4R-KO mice would be a unique model to investigate the sequence of events that make up diet-induced steatosis, liver fibrosis, and HCC.

\section{Adipose Tissue Dysfunction and Hepatic Lipid Accumulation}

The adipose tissue is primarily an energy reservoir that stores fatty acids in the form of triglyceride and an endocrine organ that secretes various hormones called adipocytokines. There is a close link between adipose tissue dysfunction and the pathophysiology of obesity-related diseases, including NAFLD/NASH. Since it is reported that macrophages infiltrate into obese adipose tissue in mice and humans [31, 32], the roles of macrophages in adipose tissue dysfunction have been intensively studied. Adipose tissue macrophages predominantly secrete proinflammatory cytokines and interact with adipocytes to induce chronic inflammation [33]. Although the balance between lipogenesis and lipolysis is tightly regulated by hormones, such as insulin and the sympathetic 
Table 1 Animal models of NAFLD and NASH

\begin{tabular}{|c|c|c|c|c|c|}
\hline Model & Induction method & Duration & Features & Histology & References \\
\hline $\begin{array}{l}\text { High-fat diet } \\
\text { (HFD) }\end{array}$ & $\begin{array}{l}\text { Diet with } 40-75 \% \text { of calory } \\
\text { intake from fat }\end{array}$ & -1 year & $\begin{array}{l}\text { Obesity, insulin } \\
\text { resistance, } \\
\text { dyslipidemia }\end{array}$ & $\begin{array}{l}\text { Steatosis, mild } \\
\text { inflammation (hepatic } \\
\text { crown-like structure } \\
(\text { CLS)) and fibrosis }\end{array}$ & $15-17$ \\
\hline $\begin{array}{l}\text { High-fat high- } \\
\text { cholesterol diet }\end{array}$ & $\begin{array}{l}\text { Diet with high content of } \\
\text { fat }(40-75 \%) \text { and } \\
\text { cholesterol }(0.1-2 \%) \pm \\
\text { cholic acid }\end{array}$ & $12-24$ weeks & $\begin{array}{l}\text { Weight gain, } \\
\text { insulin resistance, } \\
\text { dyslipidemia }\end{array}$ & $\begin{array}{l}\text { Steatosis, } \\
\text { inflammation (hepatic } \\
\text { CLS), fibrosis, } \\
\text { ballooning }\end{array}$ & $18-20$ \\
\hline $\begin{array}{l}\text { Methionine } \\
\text { choline-deficient } \\
\text { diet }\end{array}$ & $\begin{array}{l}\text { Diet deficient in methionine } \\
\text { and choline }\end{array}$ & $\begin{array}{l}3 \text { weeks steatohepatitis, } \\
5-8 \text { weeks fibrosis }\end{array}$ & $\begin{array}{l}\text { Weight loss, insulin } \\
\text { sensitive, low blood } \\
\text { glucose and } \\
\text { triglyceride levels }\end{array}$ & $\begin{array}{l}\text { Steatosis, } \\
\text { inflammation (hepatic } \\
\text { CLS), fibrosis }\end{array}$ & $21-23$ \\
\hline $\begin{array}{l}\text { Choline-deficient, } \\
\text { amino acid-- } \\
\text { defined diet } \\
\text { combined with } \\
\text { HFD }\end{array}$ & $\begin{array}{l}\text { Diet deficient in choline } \\
\text { and mild restriction of L- } \\
\text { amino acid with high } \\
\text { content of fat }\end{array}$ & $\begin{array}{l}8-12 \text { weeks } \\
\text { steatohepatitis, 16-24 } \\
\text { weeks steatohepatitis } \\
\text { and fibrosis, } \geq 30 \text { weeks } \\
\text { HCC }\end{array}$ & $\begin{array}{l}\text { Weight gain, } \\
\text { insulin resistance }\end{array}$ & $\begin{array}{l}\text { Steatosis, } \\
\text { inflammation (hepatic } \\
\text { CLS), fibrosis, } \\
\text { ballooning }\end{array}$ & 24 \\
\hline$f_{o z} / f_{o z}$ mice & $\begin{array}{l}\text { Alms } 1 \text { mutant mice fed an } \\
\text { atherogenic diet ( } 40 \% \text { fat, } \\
34 \% \text { sucrose, } 0.2 \% \\
\text { cholesterol) }\end{array}$ & $\begin{array}{l}16 \text { weeks steatohepatitis, } \\
24-36 \text { weeks fibrosis, } \\
\geq 52 \text { weeks HCC }\end{array}$ & $\begin{array}{l}\text { Obesity, insulin } \\
\text { resistance, } \\
\text { dyslipidemia }\end{array}$ & $\begin{array}{l}\text { Steatosis, } \\
\text { inflammation (hepatic } \\
\text { CLS), fibrosis, } \\
\text { ballooning }\end{array}$ & 27 \\
\hline MC4R-KO mice & $\begin{array}{l}\text { MC4R deficient mice fed } \\
\text { HFD or an atherogenic diet } \\
\text { ( } 40 \% \text { fat, } 34 \% \text { sucrose, } \\
0.2 \% \text { cholesterol) }\end{array}$ & $\begin{array}{l}8 \text { weeks steatohepatitis, } \\
16-24 \text { weeks fibrosis, } \\
\geq 48 \text { weeks HCC }\end{array}$ & $\begin{array}{l}\text { Obesity, insulin } \\
\text { resistance, } \\
\text { dyslipidemia }\end{array}$ & $\begin{array}{l}\text { Steatosis, } \\
\text { inflammation (hepatic } \\
\text { CLS), fibrosis, } \\
\text { ballooning }\end{array}$ & $6,28,29$ \\
\hline
\end{tabular}

nervous system, chronic inflammation induces insulin resistance, which lead to increased fatty acid release from the adipose tissue [34, 35]. Proinflammatory cytokines also stimulate lipolysis in adipocytes and induce dysregulation of adipocytokine production (Fig. 1). Moreover, macrophages foam a unique histological structure termed crown-like structure (CLS), where CD11-positive macrophages surround dead or dying adipocytes to engulf the cell debris and residual lipids [36]. Tanaka et al. reported that macrophage-inducible C-type lectin, which is expressed in CLS-constituting macrophages, is a novel regulator of adipose tissue fibrosis [37]. Several lines of evidence have been accumulated that fibrotic changes in the adipose tissue limit adipocyte expandability and lipid storage capacity, leading to ectopic lipid accumulation in the liver and other organs (Fig. 1) [38]. Adipose tissue fibrosis and limited expansion were observed in rodent models of NASH prior to developing hepatic phenotypes [6, 27], suggesting that fatty liver disease becomes overt when adipose tissue cannot meet the demand of excessive lipid storage in addition to the dysregulation of adipocytokine production.

\section{Hepatocyte Death-induced Inflammation and Liver Fibrosis}

The "two-hit hypothesis" or the more recent "multiple parallel-hit hypothesis" has been proposed as the molecular mechanisms for the progression from simple steatosis [39, 40]. Both hypotheses implicate lipid accumulation in hepatocytes and inflammatory cues. In contrast to benign simple hepatic steatosis with triglyceride accumulation, NASH is characterized by accumulated lipotoxic fat, such as cholesterol, diacylglycerol, fatty acids, and phospholipids, leading to hepatocyte injury and death [41]. Therefore, an increased hepatocyte death rate is a prominent feature that differentiates NASH from simple steatosis [42]. There exist at least two distinct subsets of macrophages in the liver, resident Kupffer cells and recruited macrophages derived from circulating monocytes. Since macrophages are the principal phagocytic cells in the liver, it is conceivable that interactions between dead hepatocytes and macrophages contribute to fibrogenic changes in NASH development. Using our NASH model, we found hepatic CLS in the livers of MC4R-KO mice, in which CD11c-positive macrophages surround dead or dying hepatocytes with large lipid droplets (Fig. 2) [43, 44]. Activated fibroblasts and 


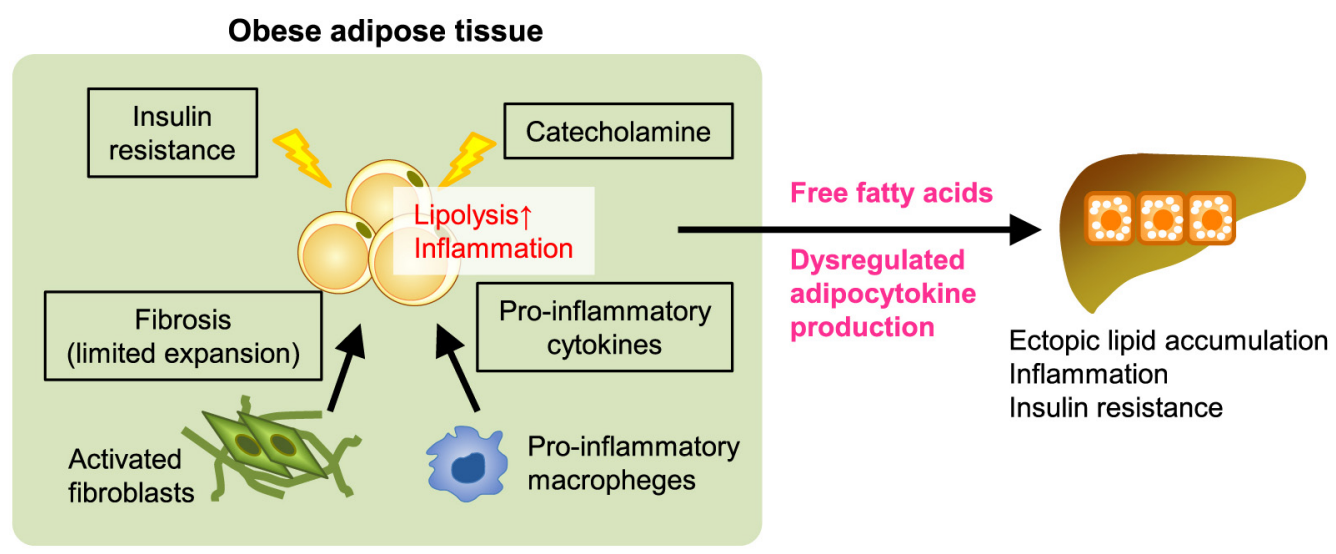

Fig. 1 Adipose tissue inflammation and ectopic lipid accumulation

Macrophages infiltrate into obese adipose tissue, and enhance the inflammatory changes through the crosstalk with mature adipocytes. Adipose tissue inflammation results in increased lipolysis and dysregulation of adipocytokine production.

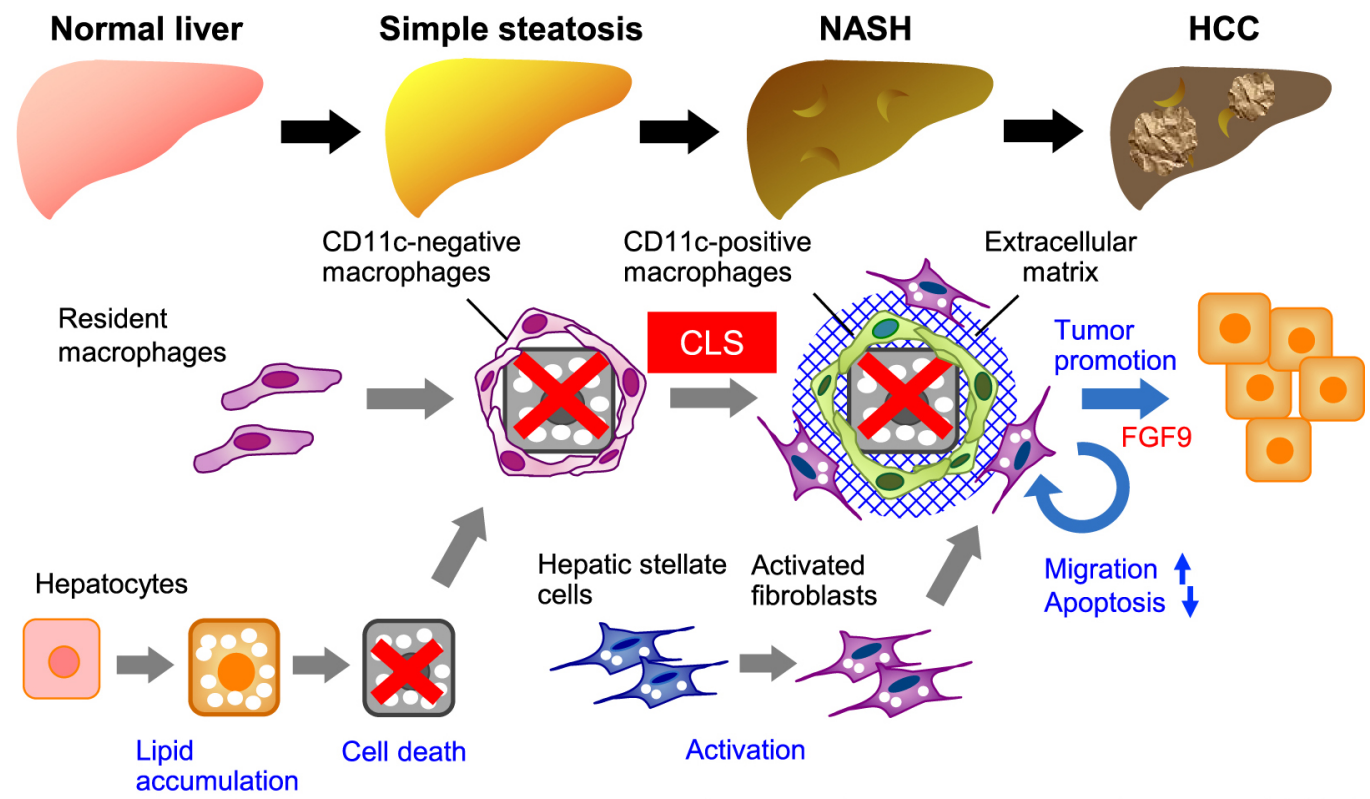

Reproduced with permission from Asakawa et al. (2019) Sci Rep 9:19601 [52].

Fig. 2 Potential role of CD11c-positive macrophages and activated fibroblasts in the progression from hepatic steatosis to NASH and HCC.

During the development of NASH, resident macrophages aggregate around dying or dead hepatocytes to constitute CLS, and these macrophages become CD11c-positive in response to hepatocyte death, with unique gene expression profiles. Metabolic stress such as saturated fatty acids induces FGF9 expression in activated fibroblasts, thereby increasing cell migration and viability of fibroblasts and hepatoma cells, and thus promoting tumor growth.

collagen deposition are observed near hepatic CLS, and the number of hepatic CLS correlates with the extent of liver fibrosis [43]. Given that hepatic CLS formation precedes fibrosis development in MC4R-KO mice, they could be a prognostic marker for NASH development. Hepatic CLS is observed in other NASH models, such as long-term HFD or WD feeding models and MCD diet feeding models $[25,26,43,45]$, suggesting that hepatic CLS is a common histological feature for NASH.
Notably, CD11c-positive hepatic CLS was observed in human NASH and the hepatic CLS number was positively associated with the score for ballooning degeneration, which is a hallmark for hepatocyte injury $[43,44]$. These clinical findings are consistent with our experimental data showing that hepatocyte death triggers hepatic CLS formation, thereby inducing inflammation and fibrosis in the liver. 


\section{Disease Specific Macrophage Subsets}

Since macrophages are highly heterogeneous in their origins and activation status, it is essential to reveal the subset responsible for the disease development in each etiology. Previous reports showed that recruited macrophages $\left(\mathrm{F} 4 / 80^{\text {lo }} \mathrm{CD} 11 \mathrm{~b}{ }^{\text {hi }}\right)$ play important roles in experimental models of liver fibrosis [45, 46]. On the other hand, we demonstrated that $\mathrm{F} 4 / 80^{\text {hi }} \mathrm{CD} 11 \mathrm{~b}^{\text {lo }}$ tissue resident macrophages are a major cellular source of hepatic CLS, and they turn into CD11c-positive in response to dead hepatocytes (Fig. 2) [44]. Depletion of CD11cpositive macrophages abolished CLS formation and fibrogenic changes in MC4R-KO mice. We also found that $\mathrm{CD} 11 \mathrm{c}-$ positive macrophages exhibit gene expression profiles distinct from CD11c-negative ones scattered in the liver, which were not categorized as typical M1 and M2 macrophages [44]. These findings suggest that CD11c-positive macrophages would be a NASHspecific macrophage subset that drives metabolic stressinduced liver fibrosis. Recently, several reports showed the molecular definition of macrophage subsets in fibrotic livers from mice and humans by single-cell RNA sequencing, and identified macrophages associated with increased lipid burden and tissue scarring characterized by unique surface marker expression (Trem2, Cd9, Spp1) [47-49]. Remmarie et al. revealed cell- and diseasespecific enhancer landscapes that explain the emergence of NASH-specific macrophage phenotypes [49]. A better understanding of the upstream signals and regulatory mechanisms that drive macrophage subset generation is crucial for developing novel diagnostic and therapeutic strategies.

\section{Characterization of Activated Fibroblasts in NASH}

Hepatic stellate cells (HSCs) transdifferentiate into activated fibroblasts or myofibroblasts during fibrogenesis caused by chronic inflammation. Activated fibroblasts are characterized by proliferative, migratory, inflammatory, and extracellular matrix-producing phenotypes $[50,51]$. The pathogenic role of profibrotic factors has been examined mainly in liver fibrosis models without obesity or HCC development. However, HSCs are activated by interactions with hepatocytes and other stromal cells, and it is important to understand differential activation status in each etiology and microenvironment. We have compared activated fibroblasts isolated from the liver of MC4R-KO mice fed WD for 20 weeks and those from chemically induced liver fibrosis using carbon tetrachloride as a model without obesity and HCC development [52]. Although fibrogenic pathways were activated in both types of fibroblasts, cancer-associated pathways were specifically upregulated in NASH fibroblasts at the time point when MC4R-KO mice develop only NASH [52]. We also showed that fibroblast growth factor 9 (FGF9), which is the most upregulated gene in cancerassociated pathways, was transcriptionally induced by metabolic stress such as saturated fatty acids. Moreover, FGF9 increases cell migration and viability in fibroblasts and hepatoma cells in vitro, promoting tumor growth in an in vivo xenograft model (Fig. 2) [52]. Numerous studies have demonstrated that cancer-associated fibroblasts have prominent roles in cancer pathogenesis by creating the tumor microenvironment [53]. Our observations indicate that activated fibroblasts in NASH exhibit proinflammatory and profibrogenic properties and provide the microenvironment promoting tumor progression.

\section{Conclusions}

Considerable evidence has shown that parenchymal cell injury or death is a key trigger of subsequent inflammation and tissue fibrosis, and macrophages are heterogeneous in their origin and activation status. This review discussed the recent progress in understanding how cell death induces chronic inflammation and fibrosis in the adipose tissue and the liver. CLS is the site of crosstalk between parenchymal and stromal cells, including macrophages and fibroblasts, suggesting that CLS is a driving force in developing tissue fibrosis. As hepatic CLS formation precedes NASH development, hepatic CLS could be a histological marker to reflect the progression from hepatic steatosis to NASH and a novel therapeutic target to implement preemptive medicine.

\section{Acknowledgements}

This work was supported by Grants-in-Aid for Scientific Research from the Ministry of Education, Culture, Sports, Science and Technology of Japan (19K07474), and research grants from Takeda Science Foundation, TERUMO Life Science Foundation, MSD Life Science Foundation.

\section{Disclosure}

None of the authors have any potential conflicts of interest associated with this research. 


\section{References}

1. Chalasani N, Younossi Z, Lavine JE, Charlton M, Cusi K, et al. (2018) The diagnosis and management of nonalcoholic fatty liver disease: practice guidance from the American Association for the Study of Liver Diseases. Hepatology 67: 328-357.

2. Younossi Z, Tacke F, Arrese M, Chander Sharma B, Mostafa I, et al. (2019) Global perspectives on nonalcoholic fatty liver disease and nonalcoholic steatohepatitis. Hepatology 69: 2672-2682.

3. Brunt EM, Wong VW, Nobili V, Day CP, Sookoian S, et al. (2015) Nonalcoholic fatty liver disease. Nat Rev Dis Primers 1: 15080.

4. Younossi Z, Stepanova M, Ong JP, Jacobson IM, Bugianesi E, et al. (2019) Nonalcoholic steatohepatitis is the fastest growing cause of hepatocellular carcinoma in liver transplant candidates. Clin Gastroenterol Hepatol 17: 748-755 e3.

5. Machado MV, Diehl AM (2016) Pathogenesis of nonalcoholic steatohepatitis. Gastroenterology 150: 1769-1777.

6. Itoh M, Suganami T, Nakagawa N, Tanaka M, Yamamoto $\mathrm{Y}$, et al. (2011) Melanocortin 4 receptor-deficient mice as a novel mouse model of nonalcoholic steatohepatitis. Am J Pathol 179: 2454-2463.

7. Chalasani N, Younossi Z, Lavine JE, Diehl AM, Brunt EM, et al. (2012) The diagnosis and management of nonalcoholic fatty liver disease: practice guideline by the American Gastroenterological Association, American Association for the Study of Liver Diseases, and American College of Gastroenterology. Gastroenterology 142: 1592-1609.

8. Rinella ME, Sanyal AJ (2016) Management of NAFLD: a stage-based approach. Nat Rev Gastroenterol Hepatol 13: 196-205.

9. Ekstedt M, Hagstrom H, Nasr P, Fredrikson M, Stal P, et al. (2015) Fibrosis stage is the strongest predictor for disease-specific mortality in NAFLD after up to 33 years of follow-up. Hepatology 61: 1547-1554.

10. Angulo P, Kleiner DE, Dam-Larsen S, Adams LA, Bjornsson ES, et al. (2015) Liver fibrosis, but no other histologic features, Is associated with long-term outcomes of patients with nonalcoholic fatty liver disease. Gastroenterology 149: 389-397.

11. Vilar-Gomez E, Calzadilla-Bertot L, Wai-Sun Wong V, Castellanos M, Aller-de la Fuente R, et al. (2018) Fibrosis severity as a determinant of cause-specific mortality in patients with advanced nonalcoholic fatty liver disease: a multi-national cohort study. Gastroenterology 155: 443457.e17.

12. Anstee QM, Targher G, Day CP (2013) Progression of NAFLD to diabetes mellitus, cardiovascular disease or cirrhosis. Nat Rev Gastroenterol Hepatol 10: 330-344.

13. Younossi ZM, Golabi P, de Avila L, Paik JM, Srishord M, et al. (2019) The global epidemiology of NAFLD and NASH in patients with type 2 diabetes: a systematic review and meta-analysis. J Hepatol 71: 793-801.

14. Eslam M, Newsome PN, Sarin SK, Anstee QM, Targher G, et al. (2020) A new definition for metabolic dysfunction-associated fatty liver disease: an international expert consensus statement. J Hepatol 73: 202-209.

15. Ito M, Suzuki J, Tsujioka S, Sasaki M, Gomori A, et al. (2007) Longitudinal analysis of murine steatohepatitis model induced by chronic exposure to high-fat diet. Hepatol Res 37: 50-57.

16. Nishikawa S, Yasoshima A, Doi K, Nakayama H, Uetsuka $\mathrm{K}$ (2007) Involvement of sex, strain and age factors in high fat diet-induced obesity in C57BL/6J and BALB/cA mice. Exp Anim 56: 263-272.

17. Carmiel-Haggai M, Cederbaum AI, Nieto N (2005) A high-fat diet leads to the progression of non-alcoholic fatty liver disease in obese rats. FASEB J 19: 136-138.

18. Vergnes L, Phan J, Strauss M, Tafuri S, Reue K (2003) Cholesterol and cholate components of an atherogenic diet induce distinct stages of hepatic inflammatory gene expression. J Biol Chem 278: 42774-42784.

19. Matsuzawa N, Takamura T, Kurita S, Misu H, Ota T, et al. (2007) Lipid-induced oxidative stress causes steatohepatitis in mice fed an atherogenic diet. Hepatology 46: 1392-1403.

20. Savard C, Tartaglione EV, Kuver R, Haigh WG, Farrell GC, et al. (2013) Synergistic interaction of dietary cholesterol and dietary fat in inducing experimental steatohepatitis. Hepatology 57: 81-92.

21. Weltman MD, Farrell GC, Liddle C (1996) Increased hepatocyte CYP2E1 expression in a rat nutritional model of hepatic steatosis with inflammation. Gastroenterology 111: 1645-1653.

22. Gao D, Wei C, Chen L, Huang J, Yang S, et al. (2004) Oxidative DNA damage and DNA repair enzyme expression are inversely related in murine models of fatty liver disease. Am J Physiol Gastrointest Liver Physiol 287: G1070-G1077.

23. Leclercq IA, Farrell GC, Field J, Bell DR, Gonzalez FJ, et al. (2000) CYP2E1 and CYP4A as microsomal catalysts of lipid peroxides in murine nonalcoholic steatohepatitis. $J$ Clin Invest 105: 1067-1075.

24. Ibrahim SH, Hirsova P, Malhi H, Gores GJ (2016) Animal models of nonalcoholic steatohepatitis: eat, delete, and inflame. Dig Dis Sci 61: 1325-1336.

25. Wolf MJ, Adili A, Piotrowitz K, Abdullah Z, Boege Y, et al. (2014) Metabolic activation of intrahepatic CD8+ T cells and NKT cells causes nonalcoholic steatohepatitis and liver cancer via cross-talk with hepatocytes. Cancer Cell 26: 549-564.

26. Farrell G, Schattenberg JM, Leclercq I, Yeh MM, Goldin $\mathrm{R}$, et al. (2019) Mouse models of nonalcoholic steatohepatitis: toward optimization of their relevance to human nonalcoholic steatohepatitis. Hepatology 69: 2241-2257.

27. Larter CZ, Yeh MM, Van Rooyen DM, Teoh NC, Brooling 
J, et al. (2009) Roles of adipose restriction and metabolic factors in progression of steatosis to steatohepatitis in obese, diabetic mice. J Gastroenterol Hepatol 24: 16581668.

28. Hasenour CM, Kennedy AJ, Bednarski T, Trenary IA, Eudy BJ, et al. (2020) Vitamin E does not prevent Western diet-induced NASH progression and increases metabolic flux dysregulation in mice. J Lipid Res 61: 707-721.

29. Wang X, Zheng Z, Caviglia JM, Corey KE, Herfel TM, et al. (2016) Hepatocyte TAZ/WWTR1 promotes inflammation and fibrosis in nonalcoholic steatohepatitis. Cell Metab 24: 848-862.

30. Balthasar N, Dalgaard LT, Lee CE, Yu J, Funahashi H, et al. (2005) Divergence of melanocortin pathways in the control of food intake and energy expenditure. Cell 123: 493-505.

31. Weisberg SP, McCann D, Desai M, Rosenbaum M, Leibel $\mathrm{RL}$, et al. (2003) Obesity is associated with macrophage accumulation in adipose tissue. J Clin Invest 112: 17961808.

32. Xu H, Barnes GT, Yang Q, Tan G, Yang D, et al. (2003) Chronic inflammation in fat plays a crucial role in the development of obesity-related insulin resistance. J Clin Invest 112: 1821-1830.

33. Tanaka M, Itoh M, Ogawa Y, Suganami T (2018) Molecular mechanism of obesity-induced 'metabolic' tissue remodeling. J Diabetes Investig 9: 256-261.

34. Reilly SM, Saltiel AR (2017) Adapting to obesity with adipose tissue inflammation. Nat Rev Endocrinol 13: 633643.

35. Lumeng CN, Saltiel AR (2011) Inflammatory links between obesity and metabolic disease. J Clin Invest 121 : 2111-2117.

36. Lumeng CN, Bodzin JL, Saltiel AR (2007) Obesity induces a phenotypic switch in adipose tissue macrophage polarization. $J$ Clin Invest 117: 175-184.

37. Tanaka M, Ikeda K, Suganami T, Komiya C, Ochi K, et al. (2014) Macrophage-inducible C-type lectin underlies obesity-induced adipose tissue fibrosis. Nat Commun 5: 4982.

38. Crewe C, An YA, Scherer PE (2017) The ominous triad of adipose tissue dysfunction: inflammation, fibrosis, and impaired angiogenesis. J Clin Invest 127: 74-82.

39. Day CP, James OF (1998) Steatohepatitis: a tale of two "hits"? Gastroenterology 114: 842-845.

40. Tilg H, Moschen AR (2010) Evolution of inflammation in nonalcoholic fatty liver disease: the multiple parallel hits hypothesis. Hepatology 52: 1836-1846.

41. Puri P, Baillie RA, Wiest MM, Mirshahi F, Choudhury J, et al. (2007) A lipidomic analysis of nonalcoholic fatty liver disease. Hepatology 46: 1081-1090.

42. Feldstein AE, Canbay A, Angulo P, Taniai M, Burgart LJ, et al. (2003) Hepatocyte apoptosis and fas expression are prominent features of human nonalcoholic steatohepatitis. Gastroenterology 125: 437-443.

43. Itoh M, Kato H, Suganami T, Konuma K, Marumoto Y, et al. (2013) Hepatic crown-like structure: a unique histological feature in non-alcoholic steatohepatitis in mice and humans. PLoS One 8: e82163.

44. Itoh M, Suganami T, Kato H, Kanai S, Shirakawa I, et al. (2017) CD11c+ resident macrophages drive hepatocyte death-triggered liver fibrosis in a murine model of nonalcoholic steatohepatitis. JCI Insight 2: e92902.

45. Miura K, Yang L, van Rooijen N, Ohnishi H, Seki E (2012) Hepatic recruitment of macrophages promotes nonalcoholic steatohepatitis through CCR2. Am J Physiol Gastrointest Liver Physiol 302: G1310-G1321.

46. Seki E, de Minicis S, Inokuchi S, Taura K, Miyai K, et al. (2009) CCR2 promotes hepatic fibrosis in mice. Hepatology 50: 185-197.

47. Ramachandran P, Dobie R, Wilson-Kanamori JR, Dora EF, Henderson BEP, et al. (2019) Resolving the fibrotic niche of human liver cirrhosis at single-cell level. Nature 575: 512-518.

48. Remmerie A, Martens L, Thone T, Castoldi A, Seurinck $\mathrm{R}$, et al. (2020) Osteopontin expression identifies a subset of recruited macrophages distinct from kupffer cells in the fatty liver. Immunity 53: 641-657.e14.

49. Seidman JS, Troutman TD, Sakai M, Gola A, Spann NJ, et al. (2020) Niche-specific reprogramming of epigenetic landscapes drives myeloid cell diversity in nonalcoholic steatohepatitis. Immunity 52: 1057-1074.e7.

50. Tsuchida T, Friedman SL (2017) Mechanisms of hepatic stellate cell activation. Nat Rev Gastroenterol Hepatol 14: 397-411.

51. Burt AD (1999) Pathobiology of hepatic stellate cells. $J$ Gastroenterol 34: 299-304.

52. Asakawa M, Itoh M, Suganami T, Sakai T, Kanai S, et al. (2019) Upregulation of cancer-associated gene expression in activated fibroblasts in a mouse model of non-alcoholic steatohepatitis. Sci Rep 9: 19601.

53. Kalluri R (2016) The biology and function of fibroblasts in cancer. Nat Rev Cancer 16: 582-598. 